ARTICLE

\title{
High-resolution vector microwave magnetometry based on solid-state spins in diamond
}

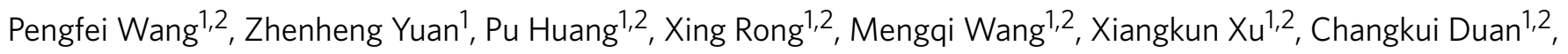
Chenyong Ju, ${ }^{1,2}$, Fazhan $\mathrm{Shi}^{1,2} \&$ Jiangfeng $\mathrm{Du}^{1,2}$

The measurement of the microwave field is crucial for many developments in microwave technology and related applications. However, measuring microwave fields with high sensitivity and spatial resolution under ambient conditions remains elusive. In this work, we propose and experimentally demonstrate a scheme to measure both the strength and orientation of the microwave magnetic field by utilizing the quantum coherent dynamics of nitrogen vacancy centres in diamond. An angular resolution of $5.7 \mathrm{mrad}$ and a sensitivity of $1.0 \mu \mathrm{THz}^{-1 / 2}$ are achieved at a microwave frequency of $2.6000 \mathrm{GHz}$, and the microwave magnetic field vectors generated by a copper wire are precisely reconstructed. The solid-state microwave magnetometry with high resolution and wide frequency range that can work under ambient conditions proposed here enables unique potential applications over other state-of-art microwave magnetometry.

\footnotetext{
${ }^{1}$ Hefei National Laboratory for Physics Sciences at the Microsacle and Department of Modern Physics, University of Science and Technology of China, Hefei 230026, China. ${ }^{2}$ Synergetic Innovation Centre of Quantum Information and Quantum Physics, University of Science and Technology of China, Hefei, Anhui 230026, China. Correspondence and requests for materials should be addressed to J.D. (email: djf@ustc.edu.cn).
} 
R ecent advantages in microwave (MW) technology have led to tremendous developments in communications, highspeed electronics and magnetic resonance. Most of these developments rely on the discovery of nanoscale, high-frequency MW processes $^{1-3}$ and materials ${ }^{4-6}$. Measurement of MW electromagnetic field with high spatial resolution is crucial but has been a long-standing problem. Measurements with high sensitivity and angular resolution have been achieved for MW electric field ${ }^{7,8}$. It is not so for the measurement of MW magnetic field vector, despite its importance, for example, in MW antenna test $^{9}$ and the study of the MW response of materials to the magnetic field ${ }^{5}$. Although various approaches, such as coplanar-waveguide-type probe ${ }^{10-14}$, superconducting quantum interference devices ${ }^{15}$, cold atoms ${ }^{16,17}$ or spin hall effect films ${ }^{18}$, have been applied to measure the MW magnetic field, the sensor size, the extreme measurement conditions required or the lack of vector measurement ability strongly limit their applications.

In this work, we demonstrate a high-resolution vectorial MW magnetometry based on nitrogen vacancy $(\mathrm{NV})$ centres in diamond. The NV centre is a defect that consists of a substitutional nitrogen atom and an adjacent vacancy in the carbon lattice of diamond. The ground spin triplet state of negatively charged NV centres can be initialized and measured by laser illumination and fluorescence intensity under laser illumination, respectively. Owing to its good spin properties, the NV centre has been shown to be a perfect solid-state quantum system for sensing magnetic fields with nanoscale resolution and high sensitivity under ambient conditions ${ }^{19,20}$. In previous works, NV centres have been utilized in the measurement of oscillating magnetic fields with frequencies ranging from kilohert ${ }^{19}$ to megahert $z^{21,22}$. Here we propose and then demonstrate experimentally a different scheme based on $\mathrm{NV}$ centres to realize the measurement of the vector of the MW magnetic field. The angular resolution and sensitivity are then analysed.

\section{Results}

Principles of vector MW magnetometry. The main idea of the MW magnetometry is based on the Rabi oscillation of a solidstate spin driven under resonant MW magnetic field. The Hamiltonian of the NV's electron spin is $H_{0}=\Delta S_{\mathrm{z}}^{2}+\gamma B_{0} S_{\mathrm{z}}+$ $\gamma \mathbf{B}_{\mathrm{mw}}(t) \cdot \mathbf{S}$, where $\Delta=2.87 \mathrm{GHz}$ is the zero field splitting, $\gamma=2.8025 \mathrm{MHz}$ Gauss $^{-1}$ is the gyromagnetic ratio of the electron spin, $\mathbf{S}$ and $S_{\mathrm{z}}$ are the associated spin operator and its $\mathrm{z}$ component, and $\mathbf{B}_{\mathrm{mw}}(t)=\mathbf{B}_{\mathrm{mw}} \cdot \cos (2 \pi f t)$ is the MW magnetic field. We selectively address the spin operation on transition of $m_{s}=0 \leftrightarrow-1$ (denoted as $|0\rangle$ and $|-1\rangle$, respectively) with a transition frequency of $\omega_{0}=\Delta-\gamma B_{0}$ (Fig. 1b). Then the system can be treated similarly as spin $1 / 2$. The resonance condition $\omega_{0}=f$ can be fulfilled by adjusting $B_{0}$. The component of the vector $\mathbf{B}_{\mathrm{mw}}$ that perpendicular to $S_{\mathrm{z}}$, denoted as $\mathbf{B}_{\mathrm{mwp}}$, can be treated as the sum of two circular polarized field with opposite rotating directions. The left-hand rotating circular polarized field $B_{1}$ with an amplitude of $B_{1}=\frac{\sqrt{2}}{2} B_{\text {mwp }}$ directly drives the spin rotation (Fig. 1c). The Hamiltonian $H_{0}$ can be simplified in the rotation frame as $H_{r}=\gamma B_{1} S_{x}$ (Fig. 1d), which results in the spin state oscillating between $|0\rangle$ and $|-1\rangle$ with Rabi frequency $\Omega_{r}=\gamma B_{1}$. In the Bloch sphere representation, the spin state vector rotates around $\mathbf{B}_{1}$ by an angle of $\alpha=2 \pi \gamma B_{1} \tau$ after a sensing time $\tau$.

Decoherence of the NV spin states needs to be taken into account in an actual case. After considering the interaction between $\mathrm{NV}$ and the ${ }^{14} \mathrm{~N}$ nuclear spin and the noise due to ${ }^{13} \mathrm{C}$ nuclear spin bath, we have the whole Hamiltonian $\mathrm{H}=\mathrm{H}_{0}+$ $\mathbf{S} \cdot A \cdot \mathbf{I}+Q I_{\mathrm{z}}^{2}+b S_{\mathrm{z}}$, where $\mathbf{I}$ is the ${ }^{14} \mathrm{~N}$ nuclear spin operator, $A$ is the hyperfine coupling tensor and $b$ denotes the effective

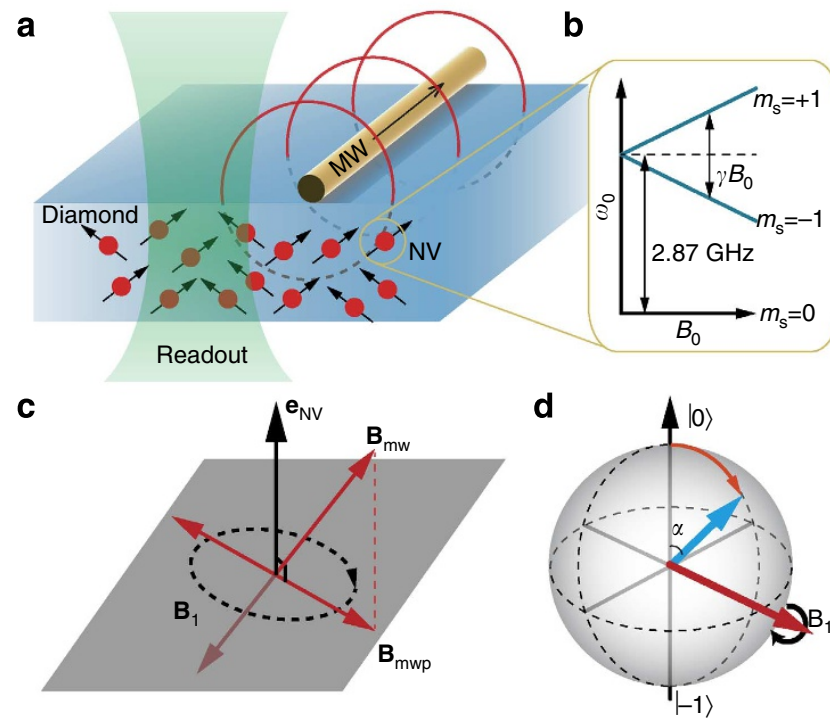

Figure 1 | Experimental setup and principle of the $\mathbf{M W}$ magnetometry.

(a) Schematic view of the setup for the demonstration of vector MW magnetometry. The 532-nm green laser is focused several micrometres below the top surface of the diamond. A copper wire of $22 \mu \mathrm{m}$ diameter above the diamond plate is used for generating the MW field. The MW passes through the copper wire and generates a linearly polarized oscillating magnetic field (red lines). (b) Zeeman splitting of the electron spin of the NV centre. (c) Schematic view of the MW magnetic field vectors and axis of the NV centre in the laboratory frame. The dark grey surface is perpendicular to the axis of the NV centre. (d) Bloch sphere view of the dynamics of the spin state.

fluctuation magnetic field of the noise ${ }^{23,24}$. There are two factors need to be considered. One is the off resonance due to the hyperfine interaction with the nitrogen nuclear spin. The probability on $|0\rangle$ under off resonance MW driving is written as ${ }^{25}$

$$
p(\omega)=\frac{1}{2}+\frac{\Omega_{r}^{2}}{2 \Omega^{2}} \cos (2 \pi \tau \Omega),
$$

where $\Omega=\sqrt{\Omega_{r}^{2}+\delta \omega^{2}}$ is the Rabi frequency under offresonance frequency detuning $\delta \omega=\omega-f$. In existence of ${ }^{14} \mathrm{~N}$ nuclear spin, the electron spin resonance transition line splits into three peaks with frequencies $f+m_{I} A$, where $m_{I}=0, \pm 1$ denotes the spin state of ${ }^{14} \mathrm{~N}$ nuclear spin (Fig. 2a). As a result, there are two frequencies $\Omega_{\text {fast }}$ and $\Omega_{\text {slow }}$ in the Rabi oscillation curve fulfilling the relation of $\Omega_{\text {fast }}^{2}=\Omega_{\text {slow }}^{2}+A^{2}$. $\Omega_{\text {slow }}$ is the Rabi frequency of the on-resonance peak and indicates $B_{1}$ value. The other factor is the inhomogeneous line broadening effect caused by the spin bath noise that dominates the signal decay. The broadened line shape can be characterized by adding a Gaussian type noise to the electron $\operatorname{spin}^{23-25}$ :

$$
P_{m_{I}}(\omega)=k_{I} \sqrt{\frac{\pi}{2}} T_{2}^{*} e^{-\left[\omega-\left(f+m_{I} A\right)\right]^{2} T_{2}^{* 2} \pi^{2}},
$$

where $k_{I}$ is the relative height of each peak and $T_{2}{ }^{*}$ is the electron spin dephasing time. The final signal can be obtained from integration of all the off-resonance contributions in the three peaks:

$$
\tilde{p}(\omega)=\sum_{m_{I}=0, \pm 1} \int_{-\infty}^{+\infty} P_{m_{I}}(\omega) p(\omega) d \omega .
$$

The magnetic field vector can be reconstructed by making use of 


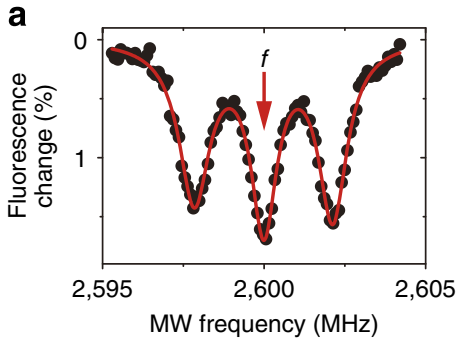

b

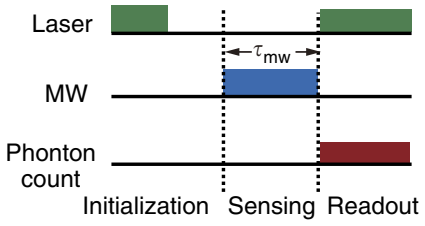

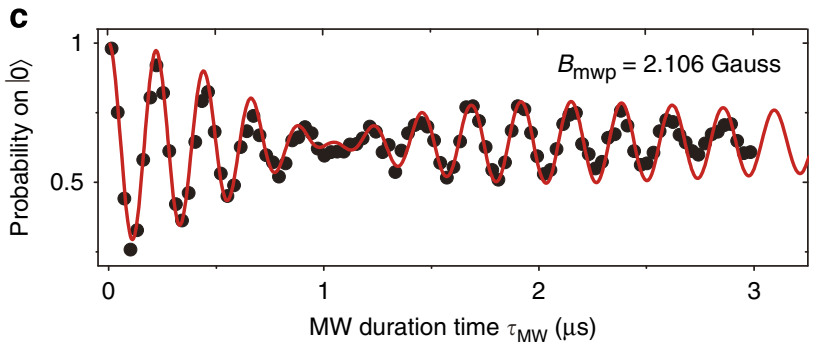

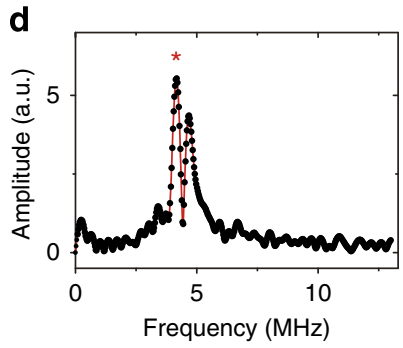

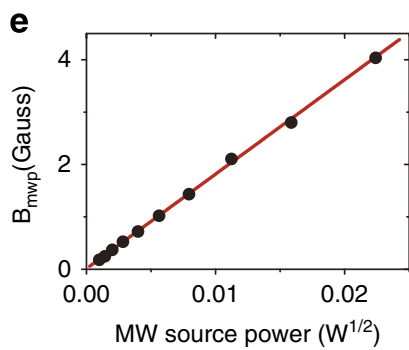

Figure 2 | Experiment results of the MW magnetic field amplitude measurement. (a) Electron spin resonance lines of the NV centres. (b) Pulse sequence of vector MW magnetometry. The 5 - $\mu$ s laser pulse and $2-\mu \mathrm{s}$ waiting time initialize the NV centre to $|0\rangle$. Then the MW is on and the NV centre sense the $\mathrm{MW}$ magnetic field. Finally, the laser is on and the amount of photons is counted to determine the spin state. (c) Rabi oscillations. Each point of the data is repeated for $1.5 \times 10^{5}$ times to increase the signal to noise ratio. The fitting yields $B_{\text {mwp }}=(2.106 \pm 0.002) \times 10^{-4} \mathrm{~T}^{*}$ and a sensitivity of $1.0 \mu \mathrm{T} \mathrm{Hz}-1 / 2$. (d) Fourier transformation of Rabi oscillations in $\mathbf{c}$, with a red line connecting the data. The frequencies of the peak $\left(^{*}\right)$ and the other one are $4.17 \mathrm{MHz}$ and 4.68 $\mathrm{MHz}$. (e) Measured projected MW magnetic field versus MW source power.

NV centres with four different orientations (Fig. 3a), [111], [-111], [1-11], [-1-11] (labelled as 1, 2, 3, 4). The static magnetic field can be aligned subsequently to each of the four orientations, and then the set of NV centres of the orientation is selectively driven and its Rabi oscillation is measured to deduce the relevant projected MW magnetic field $B_{\text {mwp }}^{i, \exp }(i=1,2,3$ and 4$)$, which is related to $\mathbf{B}_{\text {mwp }}$, as shown in Fig. 1c, by

$$
B_{\mathrm{mwp}}^{i}=\left|\mathbf{B}_{\mathrm{mw}} \times \mathbf{e}_{\mathrm{NV}}^{i}\right|(i=1,2,3 \text { and } 4) .
$$

The four relations in equation (4) are over-complete but theoretically consistent in determination all the three components of $\mathbf{B}_{\mathrm{mw}}=\left(B_{\mathrm{x}}, B_{\mathrm{y}}, B_{\mathrm{z}}\right)$. Actually, any three of them should be sufficient, except for the exceptionally few singular points such as $\mathbf{B}_{\text {mwp }}$ being along [100], [010] or [001] (Supplementary Note 1). Hence the sensor can be a nanosized diamond with three or more appropriate NV centres. For the sample with NV centres of all the four orientations used here, we can make use of the over-completeness by applying Maximum-likelihood estimation method to the data to obtain the best estimation of the magnetic vector. The likelihood can be written as

$$
\ell \propto \prod_{i=1}^{4} \exp \left\{-\frac{1}{2 \sigma_{i}^{2}}\left[B_{\mathrm{mwp}}^{i}\left(B_{x}, B_{y}, B_{z}\right)-B_{\mathrm{mwp}}^{i, \exp }\right]^{2}\right\}
$$

Maximizing $\ell$ will find the optimum value of the vector according to Bayes' theorem (Supplementary Note 4). The MW magnetic field can be uniquely determined in this way.

Experimental demonstration of the magnetometry. The probe head of the setup is shown in Fig. 1a. For the ease of demonstration, we use a chemical vapour deposition synthesized, type IIa diamond plate of $50 \mu \mathrm{m}$ in thickness from Element Six, rather than a nanodiamond that contains NV centres as the magnetometer. The average distance between each NV centre is less than $100 \mathrm{~nm}$, and there are $\sim 20 \mathrm{NV}$ centres in the laser spot. The static magnetic field $\mathbf{B}_{0}$ is aligned to the axis of each $\mathrm{NV}$ centre group to avoid ground-state mixing. The MW electronic circuits are designed to be $50 \Omega$ impedance and the total power reflection is reduced to $S_{11}<-10 \mathrm{~dB}$, so that a highly linearly polarized MW magnetic field is generated. As the relative permeability of diamond crystal is $\sim 1.0000$, the magnetic field inside the diamond should be the same as that outside of it. In our demonstration, we fix the applied static field to $B_{0} \approx 94.34$ Gauss, and the corresponding frequency for the MW field is $f=2.6000 \mathrm{GHz}$.

The pulse sequence and the measured Rabi oscillation results are shown in Fig. 2b. Figure $2 \mathrm{c}$ shows an example of Rabi oscillations under the MW source power of $0.13 \mathrm{~mW}$. By fitting the curve, we derive the projected MW magnetic field strength. $B_{\text {mwp }}=(2.106 \pm 0.002) \times 10^{-4} \mathrm{~T}$ (see Supplementary Note 2 and 3 for details). The fitting result is confirmed by the Fourier transformation of the data in the frequency domain (Fig. 2d). By varying the MW source power, we find that proportionality of the projected MW magnetic field strength deduced from the measured Rabi oscillation to the square root of the MW source power holds in a wide $B_{\text {mwp }}$ range (Fig. 2e and Supplementary Fig. 1).

Further demonstration to show the angular resolution was performed by using the vector MW magnetometry to measure in a series of space locations the strength and orientation of the linearly polarized MW magnetic field generated by a copper wire of $\sim 5 \mathrm{~mm}$ in length. The copper wire is much longer than the distance between the copper wire and the diamond surface, and so can be treated as indefinitely in length. The MW magnetic near field has a magnitude inversely proportional to the distance to the wire and an orientation along the tangent as Ampere's circuital law. We scan the laser spot in a line to measure Rabi Oscillations. The scanning line is divided into 150 points with a step of $\sim 130 \mathrm{~nm}$ and several micrometres below the diamond surface (Fig. 3b). By controlling the orientation of the static magnetic field, we measure the Rabi oscillations of the NV centres of all the four groups in each point. In prior, the diamond is put at an angle of $0.37 \mathrm{rad}$ with the copper wire to avoid the singular point (Supplementary Fig. 2). The experiment results are shown in Fig. 3c (see Supplementary Table 1 for the fitting parameters). All 
a
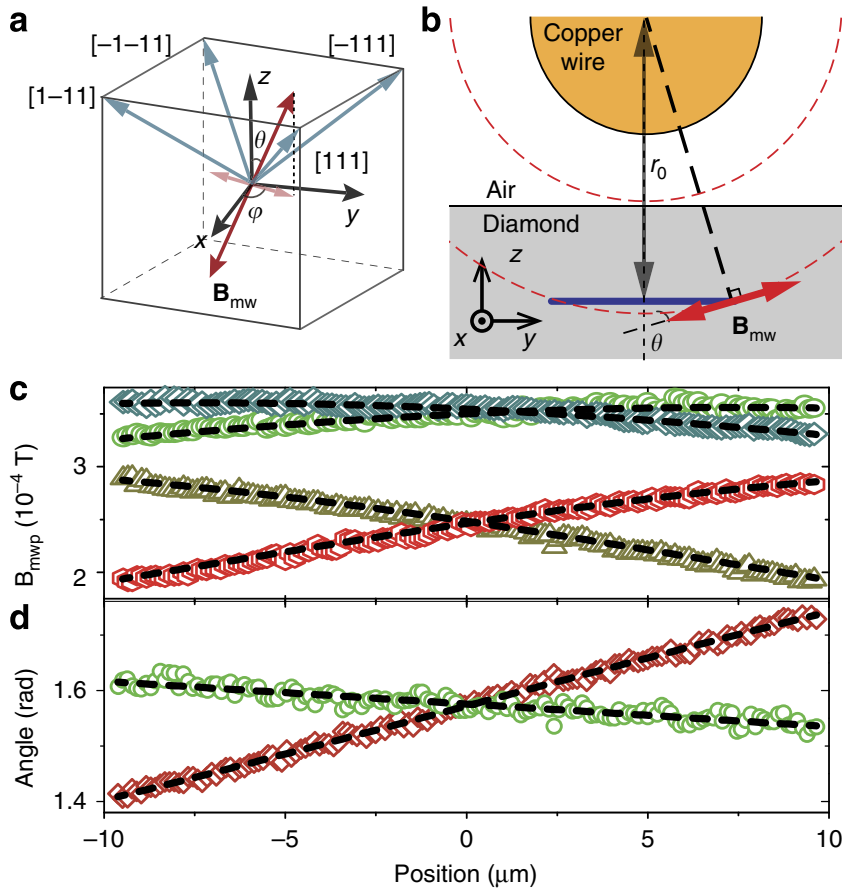

Figure 3 | Experiment results of vector MW magnetometry. (a) Definition of the vector and angle. (b) Schematic view of the MW magnetic field distribution and the scanning line. In the near field, the oscillation current in the copper wire generates a linearly polarized magnetic field with a direction perpendicular to the copper wire and along the tangent. The coordinate is set as follows: $x$ is along the copper wire, $y$ is perpendicular to the copper wire and parallel to the diamond surface, and $z$ is vertical to the diamond surface. The scanning line (black line) is made below the copper wire and along the $y$ axis. (c) Measured $B_{\text {mwp }}$ with the magnetic field along [111] (triangle), [-111] (diamond), [1-11] (rhombic) and [-1-11] (hexagon). The fitting curve to the data (dashed line) gives the distance $r_{0}=47.49 \mu \mathrm{m}$. (d) $\theta$ (diamond) and $\varphi$ (circle) calculated from the data in c. The dashed line is the fitting curve to the data. The fitting yields the angular resolution of $\delta \theta=5.7 \mathrm{mrad}$ and $\delta \varphi=9.9 \mathrm{mrad}$.

the three components of the vector $\mathbf{B}_{\mathrm{mw}}$ are then deduced by utilizing maximum-likelihood estimation method, and so the orientation of the vector is obtained (Supplementary Note 5). The orientation of $\mathbf{B}_{\mathrm{mw}}$ versus position is shown in Fig. 3d, together with theoretically predicted lines. The experiment result of $\varphi$ and $\theta$ perfectly matches the theoretical predictions of the MW magnetic field. The minimum angular resolution achieved is $\delta \omega=5.7 \mathrm{mRad}$, which can be further improved by increasing the detecting time or photon collection efficiency ${ }^{26,27}$.

\section{Discussion}

In conclusion, we have proposed a vector MW magnetometry scheme using NV centres in diamonds with high sensitivity and spatial resolution and under ambient conditions, and then carried out experimental demonstration of the amplitude and angular sensitivity with the measurement of the MW magnetic field vector generated by an infinitely long copper wire. In our demonstration, the spatial resolution is only diffraction limited, which gives a sensor size of $\sim 230 \mathrm{~nm}$ (see Methods section). The capability of the vector MW magnetometry based on the NV centre is not limited by what we demonstrate here. By adopting wide-field imaging technology, a two-dimensional reconstruction of the MW magnetic field can be realized ${ }^{28,29}$. Based on solidstate spins of NV centre in diamond, the sensor size can be reduced to ten nanometre scale by a well-treated nanodiamond ${ }^{30}$. Also the working frequency can be extended to sub-terahertz band continuously by an external static field $8.6 \mathrm{~T}$ (ref. 31) and reach terahertz under $35 \mathrm{~T}$, which is available in the current technology.

Our solid-state MW vector magnetometry will directly find its application in testing the MW electronics and materials. Owing to long-term stability of the NV centre, it can also serve for the purpose to stabilize the amplitude of the MW. Moreover, by combining the NV centres with a scanning probe microscopy, the $\mathrm{NV}$ centre-based scanning near-field vector MW magnetometry can be used in studying antenna radiation as a non-destructive measurement ${ }^{9}$. Finally, as the sensor can be downsized to nanometre scale, our scheme may promote the ferromagnetic resonance imaging ${ }^{32}$ to the nanoscale.

\section{Methods}

Experiment setup. Our setup is based on our homebuilt confocal microscopy. A temperature-stabilized diode laser (CNI MGL-III-532) is used for the initialization and readout of quantum states. The laser beam passes through a double-pass acoustic optical modulator (Crystal Technology 3200-121) to reach an isolation of about $60 \mathrm{~dB}$. Then it enters a single-mode fibre acting as a spatial filter. A high numerical aperture (NA) objective lens (Olympus UPLSAPO 100XO) is used for the laser focusing and fluorescence collection. A high-resolution scanner (Asylum research) and fast steering mirror (Newport FSM-300) is used for the laser scanning. The magnetic field is applied by a permanent magnet on a three-dimensional translation stage. The fluorescence passes through a band-pass filter with $650-775 \mathrm{~nm}$ and $20 \mu \mathrm{m}$ pinhole to the APD (Perkin Elemer SPCM-AQRH-14), which limit the optical detection size to be diffraction limited. The MW generated by a MW source (Agilent N5181B) is cut to square wave pulse by an MW PIN switch (CMCS0947A-C1, 3 ns rising time). An amplifier (Mini-circuits ZHL-16 W-43+) is used to amplify the MW signal.

Thermal drift. The experimental measurements in this demonstration last for a month, so the long-term stability of the whole setup is crucial. First, we put the whole probe head in a chamber with temperature stability of $\pm 50 \mathrm{mK}$ to reduce mechanical thermal drift, which enables us to focus on the same point for a long time without any drift. Second, we put some diamond nanocrystal with NV centres on the diamond surface for locating and carrying out position feedback. Finally, the most important thing is to keep all the MW circuit stable to $\pm 0.5 \mathrm{~K}$ and keep a constant output power, so that the MW magnetic field amplitude remains stable during the measurement.

Sensor size and spatial resolution. In our demonstration, the diamond sample is rather big, and the spatial resolution is the pinhole size divided by the magnification of the objective lens, with the low limit being the Abbe diffraction limit, which can be written as:

$$
d=\frac{\lambda}{2 \times N A}
$$

With our band-pass filter wavelength, $\lambda \sim 650 \mathrm{~nm}$, we obtain $d \sim 230 \mathrm{~nm}$. However, a well-selected nanosized diamond could be used to reach a nanoscale spatial resolution.

\section{References}

1. Hen-Sum Philip, W., Frank, D. J., Solomon, P. M., Wann, C. H. J. \& Welser, J J. Nanoscale CMOS. Proc. IEEE 87, 537-570 (1999).

2. Bernstein, K. et al. High-performance cmos variability in the $65-\mathrm{nm}$ regime and beyond. IBM J. Res. Dev. 50, 433-449 (2006).

3. Calhoun, B. H. et al. Digital circuit design challenges and opportunities in the era of nanoscale cmos. Proc. IEEE 96, 343-365 (2008).

4. Duan, X., Huang, Y., Cui, Y., Wang, J. \& Lieber, C. M. Indium phosphide nanowires as building blocks for nanoscale electronic and optoelectronic devices. Nature 409, 66-69 (2001).

5. Yen, T. J. et al. Terahertz magnetic response from artificial materials. Science 303, 1494-1496 (2004)

6. Schurig, D. et al. Metamaterial electromagnetic cloak at microwave frequencies. Science 314, 977-980 (2006).

7. Sedlacek, J. A. et al. Microwave electrometry with Rydberg atoms in a vapour cell using bright atomic resonances. Nat. Phys. 8, 819-824 (2012).

8. Sedlacek, J. A., Schwettmann, A., Kbler, H. \& Shaffer, J. P. Atom-based vector microwave electrometry using rubidium rydberg atoms in a vapor cell. Phys. Rev. Lett. 111, 063001 (2013).

9. Yaghjian, A. D. An overview of near-field antenna measurements. IEEE Trans Antennas Propag. 34, 30-45 (1986) 
10. Serov, R., Richardson, M. C. \& Burtyn, P. Probe for the measurement of magnetic fields with sub-nanosecond resolution. Rev. Sci. Instrum. 46, 886-888 (1975).

11. Gao, Y. \& Wolff, I. A new miniature magnetic field probe for measuring three-dimensional fields in planar high-frequency circuits. IEEE Trans. Microw. Theor. 44, 911-918 (1996).

12. Kohei, S., Chun-Ping, C., Zhewang, M., Anada, T. \& Thomas, D. W. P. Non-contacting electric and magnetic field probe for measuring EM fields on microwave planar circuits. In Microwave Conference, 2007. APMC 2007. Asia-Pacific 1-4 (IEEE, 2007).

13. Yingjie, G. \& Wolff, I. A miniature magnetic field probe for measuring fields in planar high-frequency circuits. In IEEE MTT-S 1159-1162 (1995).

14. Dubois, T. et al. Near-field electromagnetic characterization and perturbation of logic circuits. IEEE Trans. Instrum. Meas. 57, 2398-2404 (2008).

15. Black, R. C. et al. Imaging radio frequency fields using a scanning squid microscope. Appl. Phys. Lett. 66, 1267-1269 (1995).

16. Böhi, P., Riedel, M. F., Hänsch, T. W. \& Treutlein, P. Imaging of microwave fields using ultracold atoms. Appl. Phys. Lett. 97, 051101 (2010).

17. Ockeloen, C. F., Schmied, R., Riedel, M. F. \& Treutlein, P. Quantum metrology with a scanning probe atom interferometer. Phys. Rev. Lett. 111, 143001 (2013).

18. Vlaminck, V. et al. Mapping microwave field distributions via the spin hall effect. Appl. Phys. Lett. 101, 252406 (2012).

19. Maze, J. R. et al. Nanoscale magnetic sensing with an individual electronic spin in diamond. Nature 455, 644-647 (2008).

20. Balasubramanian, G. et al. Nanoscale imaging magnetometry with diamond spins under ambient conditions. Nature 455, 648-651 (2008).

21. de Lange, G. et al. Single-spin magnetometry with multipulse sensing sequences. Phys. Rev. Lett. 106, 080802 (2011).

22. Loretz, M., Rosskopf, T. \& Degen, C. L. Radio - frequency magnetometry using a single electron spin. Phys. Rev. Lett. 110, 017602 (2013).

23. Hanson, R., Dobrovitski, V. V., Feiguin, A. E., Gywat, O. \& Awschalom, D. D. Coherent dynamics of a single spin interacting with an adjustable spin bath. Science 330, 60-63 (2008).

24. Huang, P. et al. Observation of an anomalous decoherence effect in a quantum bath at room temperature. Nat. Commun. 2, 570 (2011).

25. Schweiger, A. \& Jeschke, G. Principles of Pulse Electron Paramagnetic Resonance (Oxford Univ., 2001).

26. Maletinsky, P. et al. A robust scanning diamond sensor for nanoscale imaging with single nitrogen-vacancy centres. Nat. Nanotech. 7, 320-324 (2012).

27. Hadden, J. P. et al. Strongly enhanced photon collection from diamond defect centers under microfabricated integrated solid immersion lenses. Appl. Phys. Lett. 97, 241901 (2010).

28. Steinert, S. et al. High sensitivity magnetic imaging using an array of spins in diamond. Rev. Sci. Instrum. 81, 043705 (2010).
29. Pham, L. M. et al. Magnetic field imaging with nitrogen-vacancy ensembles. New J. Phys. 13, 045021 (2011).

30. Chang, Y.-R. et al. Mass production and dynamic imaging of fluorescent nanodiamonds. Nat. Nanotech. 3, 284-288 (2008).

31. Takahashi, S., Hanson, R., van Tol, J., Sherwin, M. S. \& Awschalom, D. D. Quenching spin decoherence in diamond through spin bath polarization. Phys. Rev. Lett. 101, 047601 (2008).

32. Lee, I. et al. Nanoscale scanning probe ferromagnetic resonance imaging using localized modes. Nature 466, 845-848 (2010).

\section{Acknowledgements}

We thank Professor Fei Xue and Professor Xidong Wu for useful discussions. This work was supported by the National Key Basic Research Program of China (Grant No. 2013CB921800), the National Natural Science Foundation of China (Grant Nos.11227901, 31470835, 91021005 and 11275183) and the CAS, and the Fundamental Research Funds for the Central Universities.

\section{Author contributions}

P.W., Z.Y., F.S. prepared the setup and performed the experiments; P.W., X.R. built the microwave circuits; X.X. and M.W. designed and fabricated the microwave delivery device; J.D. supervised the setup and experiments. All authors discussed the results and participated in writing the manuscript.

\section{Additional information}

Supplementary Information accompanies this paper at http://www.nature.com/ naturecommunications

Competing financial interests: The authors declare competing financial interests.

Reprints and permission information is available online at http://npg.nature.com/ reprintsandpermissions/

How to cite this article: Wang, P. et al. High-resolution vector microwave magnetometry based on solid-state spins in diamond. Nat. Commun. 6:6631 doi: $10.1038 /$ ncomms7631 (2015).

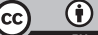

This work is licensed under a Creative Commons Attribution 4.0 International License. The images or other third party material in this article are included in the article's Creative Commons license, unless indicated otherwise in the credit line; if the material is not included under the Creative Commons license, users will need to obtain permission from the license holder to reproduce the material. To view a copy of this license, visit http://creativecommons.org/licenses/by/4.0/ 